\title{
El estudiante de medicina: sus ideas y renglones
}

DOI: $10.46932 / \operatorname{sfjdv2n2-119}$

Received in: March 1st, 2021

Accepted in: May 30th, 2021

\author{
Bernabé Ríos Nava \\ Unidad Académica de Medicina \\ Universidad Autónoma de Nayarit \\ Dirección electrónica brios1954@gmail.com \\ J. Ramón Olivo Estrada \\ Unidad Académica de Economía \\ Universidad Autónoma de Nayarit \\ Dirección electrónica olivojr@gmail.com
}

\section{RESUMEN}

Presentamos nuestra vivencia pedagógica en la unidad de aprendizaje de Socioantropología de la Salud (SaS) con los estudiantes de los grupos A y B de primer ingreso a la carrera de Médico Cirujano (ciclo escolar 2016-2017) en la Unidad Académica de Medicina (UAM) de la Universidad Autónoma de Nayarit (UAN) en su incursión a los laberintos de la lectura y escritura académicas. De nuestra intervención resaltamos el trabajo realizado durante la implementación del taller y la elaboración de la autobiografía. Nuestro propósito fue colaborar mutuamente para la comprensión y puesta en práctica de diversas habilidades, destrezas y conocimientos en la lectura y producción de textos académico-científicos.

Palabras clave estudiantes primer ingreso, escritura académica, talleres

\section{INTRODUCCIÓN}

\author{
"Raramente somos conscientes \\ de la estrecha interrelación que existe entre \\ la escritura, pensar, saber y ser". \\ Cassany, Daniel. 2006
}

Nuestra vivencia pedagógica en la unidad de aprendizaje de Socioantropología de la Salud (SaS) se llevó a cabo con los estudiantes de los grupos A y B de primer ingreso a la carrera de Médico Cirujano (ciclo escolar 2016-2017) en la Unidad Académica de Medicina (UAM) de la Universidad Autónoma de Nayarit (UAN) en su incursión a los laberintos de la lectura y escritura académicas. Resaltamos el trabajo desarrollado durante la implementación del taller y la elaboración de la autobiografía, con el propósito de colaborar mutuamente para la comprensión y puesta en práctica de diversas habilidades, destrezas y conocimientos en la lectura y producción de textos académico-científicos.

La generación 2016-2021. Se conforma por un total de 166 estudiantes, de los cuales el $46 \%$ son hombres y el $54 \%$ mujeres; a los grupos A y B pertenecen un total de 83 estudiantes (50\% del total). Sus 
rasgos demográficos son muy característicos: juventud, edad, soltería. Su presencia en los espacios universitarios les representará un cambio trascendental en todos los órdenes de su vida, porque en estos se llevará a cabo la socialización sistemática, ordenada y jerárquica en relación a la generación y transmisión de conocimientos, valores y actitudes que constituyen los ethos profesionales. (De Garay, s/f) En lo académico, las nuevas vivencias marcarán por siempre sus vidas futuras; en sus habilidades para la expresión oral y escrita, de acuerdo a diversos autores, mostrarán, por su naturaleza, diversas limitantes. Aun cuando pasaron tres años de formación preparatoriana, ésta no los dotó de lo necesario para enfrentar los nuevos desafíos. Es en la universidad donde aprenderán las estrategias para participar con suficiencia en la cultura discursiva de su disciplina mediante diversas actividades en la producción y análisis de los textos académico-científicos cuyo fin es el aprendizaje universitario. (Carlino, 2005:13)

El contexto universitario nayarita se muestra inhospito y agreste para el desarrollo de estas habilidades y conocimientos. A su falta de pericia se suman la falta de formación, lo atiborrado de los programas: "todo está bien saturado", "justo, sin espacio" y la opinión que nos hemos formado como docentes: “[...] no comprenden lo que leen”, “[...]deberían llegar al nivel superior sabiendo estudiar y escribir mejor”, “están acostumbrados a memorizar el texto” (Fernández, 2007:277). Son contadas las unidades de aprendizaje dedicadas al desarrollo de estas “[...] prácticas de lenguaje y pensamiento propías del ámbito académico superior" (alfabetización académica) (Carlino, 2005:13). En el caso de la UAM, son dos las unidades de aprendizaje que atienden estos menesteres: Desarrollo de Habilidades del Pensamiento (DHP) y Ensayo Científico (EC), las que representan el $3 \%$ del plan de estudios; además, el perfil de egreso no enfatiza las habilidades en la comunicación o expresión oral y escrita (UAM, 2017), como sí lo hacen el programa de Qfb (UACQBF, 2017) o el de Enfermería (UAE, 2017).

\section{LA UNIDAD DE APRENDIZAJE SOCIOANTROPOLOGÍA DE LA SALUD}

Como resultado de la reforma universitaria a principios del presente siglo, la UAM cambió su programa de Medicina General Integral (A-36) por el de Competencias Profesionales Integradas (CPI); en ese momento apareció SaS siendo ubicada en el primer semestre y formando parte del Tronco Básico de Área (TBA). Su propósito fue acompañar al estudiante en el estudio del fenómeno de la saludenfermedad mediante el conocimiento y la aplicación de metodologías de las ciencias sociales a fin de entender la relación y el impacto que tienen en éste las circunstancias socioeconómicas, culturales y políticas de nuestro entorno. Se constituye por cuatro grandes temas, y tiene una duración de 18 sesiones semanales de dos horas. La investigación de un tema de salud es la actividad transversal, que los estudiantes desarrollan a lo largo del ciclo escolar. 


\title{
3 NUESTRA PROPUESTA METODOLÓGICA
}

Desde el año 2015 nos dimos cuenta de la serie de problemas y limitaciones que mostraban los trabajos finales que entregaban los estudiantes, a los aspectos de limpieza se sumaban los de estructura, coherencia, cohesión, ortográfica, sintáctica. A partir de diversas pláticas grupales advertimos el escaso tiempo que sus docentes dedicaron en estas cuestiones. Ante ello decidimos contribuir a cambiar este panorama escolar y una pregunta fue nuestro eje ¿cómo incorporamos actividades de lectura y escritura académicas que fortalezcan sus habilidades y destrezas a la vez de que hacerlas atractivas?

Una empresa de esta envergadura será posible no con una materia ni con un ciclo escolar, no es una propuesta remedial, implica, “[...] que cada una de las cátedras esté dispuesta a abrir las puertas de la cultura de la disciplina que enseña para que de verdad puedan ingresar los estudiantes, que provienen de otras culturas". (Carlino, 2005:15) En tanto eso llega a nuestra realidad universitaria nayarita bien vale la pena fortalecer y difundir las experiencias que están surgiendo. Después de varias reuniones introdujimos en el mes de agosto de 2016, entre otros, los siguientes cambios en la organización del programa: se solicitaron dos trabajos de investigación (una autobiografía y un ensayo) cuyo tiempo de elaboración, de cada uno, osciló entre dos meses y medio; cada una de las 18 sesiones fue dividida en dos, en la primera se abordaron los temas del programa y la segunda fue dedicada al taller de lectura y escritura (Tle) en el cual se trabajarián los dos escritos.

\section{EL TALLER Y EL NACIMIENTO DE IDEAS-PÁRRAFOS-IDEAS}

\author{
"Me gusta el término taller por su connotación \\ artesanal porque se venga de los atracones \\ del saber y de sus modelos, porque evoca \\ el aprendizaje y la paciencia" \\ Boniface Claire, 1992. Tomado de Triogla, 2008:19
}

Nuestra concepción del Tle ha cambiado al paso de estos meses. No se constituyó tal cual (formalmente) en un principio: objetivos, actividades, tiempos y materiales (Troglia, 2008:19). La primera propuesta fue desarrollarlo en los espacios dedicados a las tutorías, lo que no funcionó por el alto ausentismo. La solución llegó al momento de abrirle un espacio en las sesiones del programa. Tenemos claro que la razón de ser del taller se basa no solo en el proceso de construcción de un texto, como proceso final, sino que a su interior hay grandes etapas muy definidas como la planificación, el proceso de creación y el resultado (Martínez, 2014:14); cada una con dinámicas y requerimientos específicos. Imaginamos al taller como el espacio que se distinguiera de las clases ordinarias: permitir la circulación del saber, la coordinación compartida, el trabajo cooperativo, propiciar el funcionamiento democrático, la participación no forzada, el humor y el juego (Troglia y Stapich, 2008:13). Lo pensamos, también, como 
el sitio donde pudiera germinar el trabajo individual en sus primeras "pinceladas" al dar respuesta a la Guía para explorar el problema retórico (Propósito, Audiencia, Autor, y Escrito) (Cassany, 1995:55), y como fase de preparación para su posterior incorporación a la "comunidad discursiva” de la disciplina; entendiendo por ello "[...] al conjunto de personas que comparten un grupo determinado de géneros discursivos" (Cassany, 2006:25).

El espíritu del Tle: a escribir se aprende escribiendo, se sustentó en la visión del enfoque metodológico "centrado en el proceso" donde la composición de los textos apuntó a generar y ordenar ideas (a pensar), hacer esquemas, revisar los borradores (pulir la estructura de la frase), corregirlos, reformularlos, en síntesis “[...]enseñar a esculpir y no enseñar esculturas” (Cassany, 1990:73). Se partió de una organización muy simple: una breve exposición del tema del día ("De lo que hay que saber para escribir bien; de las ganas de hacerlo; de lo que se puede escribir; "Accionar máquinas"; elaboración de párrafos; géneros discursivos), responder cómo el tema nos ayuda para la escritura de nuestro texto, indicaciones generales, tiempo para escribir y comentarios finales sobre los avances.

El Tle rompió con el empleo de tiempos de la sesión dedicada al programa. Se aprovechó para la conversación entre pares y con los docentes, comentar los avances, las dudas, las inquietudes, pararse y caminar por los pasillos a manera de que las ideas empezaran a fluir, sorber poco a poco el agua o el café o simplemente seleccionar del teléfono celular la música, ponerse los audifónos y continuar escribiendo "Si lo importante es aprender a comunicar[...]dejémos escribir a los alumnos en clase, para que[...]practiquen y aprendan a hacerlo[...]aprenderán[...]a reflexionar, a desarrollar ideas, a compartir, a analizar la lengua”. (Cassany, s/f) El estar al tanto del avance del trabajo y hacer los comentarios pertinentes fue una oportunidad para entender la importancia de la escritura como un proceso recursivo y etapas a las cuales atender "[...[la planificación (o preescritura), la textualización (o escritura) y la revisión (o reescritura)" (Cassany, 1999:15). ¿Llegarán a ser escritores y con ello a destacar en su disciplina? No lo sabemos, pero creemos, eso sí, mantener ese espíritu de libertad y apertura a la lectura y la escritura para todos y no olvidar que "[...] No para que todos sean artistas, sino para que nadie sea esclavo" (Rodari, 1995:8 citado por Troglia y Stapich, 2008:17).

\section{LA AUTOBIOGRAFÍA}

Se le ubica en el campo de la literatura, se dice que el autor se mueve entre lo real y lo imginario, puede anotar de más o de menos y que $[\ldots]$ "el elemento subjetivo interviene[...]Comencemos[...]a tratar las[...]autobiografías [...]como libros de literatura en los que sus autores[...]fueron poco a poco transformándolos en arte" (Avilés, 1997:49) La tomamos para trabajarla como un escrito de corte científico y porque desde nuestra visión es un (escrito-puerta) con el cual abrimos el panorama para 
conocer más adelante otros géneros académicos propios de la disciplina: el ensayo, la historia clínica, el informe de salud, "Aprender a escribir un escrito es aprender a desarrollar la práctica social correspondiente" (Cassany, 2006:21).

Además, reune varios atributos que permean la escritura y la lectura como "[...]el acto de [..]cincelar un autorretrato" (Zavala, 2013:88); hablo de mí, de mis días y años pasados, de mis metas y mis anhelos, de mis ratos de tristeza y soledad, me doy a conocer al otro y paso a la posteridad; nos acercamos a la revisión de diversos autores y sus escritos científicos; entendemos los primeros pasos de la investigación; el elaborarse de manera individual permite re-conocer sus maneras individuales de trabajo, el estilo personal de escribir, y los recursos de que disponen: el uso del diccionario, el empleo de nuevos vocablos y su significado; la construcción de la atmósfera que rodea la escritura: el espacio físico, el momento del día, tipo de vestimenta, bebidas a consumir, la iluminación y la música.

El tema central tomó las vivencias en la universidad durante los primeros dos meses y medio. Y fue acompañado de un ejercicio etnográfico al elaborar un Diario, que es una técnica empleada en la recogida de la información (Murillo, 2010:10). Las indicaciones en su elaboración fueron que se narraran las experiencias más significativas del día y de cómo ha cambiado su dinámica familiar, personal y social. Representó un hecho inédito en ellos, alguno comentó que volvía a las aulas de primaria, nos parece que “[...]resulta un material que parece sostenerse a través del tiempo[...] al paso del cambio cultural”. (Chartier, 2016)

Enfatizamos que la autobiografía tendrá un remitente y en este caso será el estudiante próximo a ingresar a la Unidad académica. Por ello entonces cabrían algunas preguntas a ser resueltas en el camino: ¿qué me gustaría comentarle? ¿cómo decirselos de la manera más clara y comprensible? Con esta consigna apuntamos a despertar su imaginación, interpelarlos y convocarlos subjetivamente. (Finocchio, 2016) Un aspecto interesante fue cuando les explicamos que su trabajo llevaría un título y ellos serían los que lo construirían. Tomamos de base el escrito de Alexia Sanz Hernández "El método biográfico en investigación social: Potencialidades y limitaciones de las fuentes orales y los documentos personales", con el propósito de unificar criterios acerca de la autobiografía. Se les dieron las orientaciones necesarias para que los trabajos finales fueran presentados impecables y su estructura cubriera los criterios de todo documento científico: tuviera cohesión y coherencia, además de aspectos de sintaxis, ortografía, redacción y evitar hasta lo más posible el plagio de información conocido bajo el término "cortar y pegar" (cut and paste) (CA White, 2009:2). Fue grata sorpresa que la totalidad de los estudiantes entregaran su trabajo final y cubrieran, en parte, las recomendaciones; aún siguieron presentándose problemas en la redacción, estructura interna, coherencia y cohesión, la ausencia de objetivos, y su poca relación con las conclusiones. 
Los títulos fueron sugestivos: Un blanco caminar por mis años preuniversitarios; Hola mundo; Serendipia; Camino hacia la meta; Maktub; Recuerdos de una vida pasada; La vida no es perfecta por sí misma, lo que sucede en ella la hace perfecta; En camino al hospital; El tren de la vida; Vocación ¿sinónimo de amor?

\section{TESTIMONIOS}

"Este curso a pesar que solo nos toca los viernes me ha sido de interés, pues hemos aprendido[...] yo en lo particular, a escribir textos de una manera más formal y correcta”, "Uno de los trabajos que me costó bastante fue la autobiografí[...]”, “[...] fue muy satisfactorio porque ya puedo hacer ensayos y trabajos siguiendo las normas APA que antes desconocía", "Me pareció una clase muy enriquecedora[...] considero que mis hábitos de lectura han mejorado y soy un mejor redactor, gracias a los conocimientos que aprendi”".

\section{CONCLUSIONES}

La composición de textos es una actividad actual, necesaria y fundamental para el ejercicio de la presente y futura práctica médica. La experiencia vivida con los estudiantes de recién ingreso nos muestra que a pesar de la afirmación anterior, el actual contexto académico se muestra adverso y árido para éste tipo de enseñanzas. Aún así, debemos insisitir en que los esfuerzos institucionales deberán de apoyar de manera decidida una formación profesional donde las habilidades para la compresión y composición de textos académico-científicos tenga un lugar preponderante a lo largo de la vida universitaria. Nuestra propuesta pedagógica deja la lección de que pueden realizarse un sinúmero de ejercicios de esta naturaleza y que los estudiantes responderán, como ha sido una constante, de manera extraordinaria; por lo que debemos continuar luchando por abrir nuevos espacios curriculares. 


\section{REFERENCIAS}

Aleza, Izquierdo Milagros. (2011). Signos ortográficos, ortotipografía y normas actuales. España: Departamento de Filología Española. Universidad de Valencia. Recuperado el 2 de Febrero de 2017, de https://www.uv.es/normas/2011/materiale s/Signos_2011.pdf

Alexopoulou, Angélica. (2010). Tipología textual y comprensión lectora en E/LE. Nebrija(9). Recuperado el 14 de Diciembre de 2016, de http://www.nebrija.com/revista-lingui stica/tipo logia-textual-ycomprension-lectora-en-e-le

Avilés, Fabila René. (1997). La autobiografía como género literario. Revista de la Universidad de México, 48-53. Recuperado el 14 de Noviembre de 2016, de http://www.revistadelauniversidad.unam.mx/ojs_rum/files/journals/1/articles/14586/ public/1458619984-1-PB.pdf

Carlino, P. (2005). Escribir, leer y aprender en la universidad. Una introduccion a la alfabetización académica. México: Fondo de Cultura Económica.

Culebras y Vives Cecilia. (2004). Taller de ortografía y redacción básicas. Cuernavaca, Morelos, México: Departamento de Desarrollo Académico e Idiomas. Seminario de investigación CE NIDET. Recuperado el 15 de Octubre de 2016, de http://ww w.cenidet.edu.mx/subaca/w eb-dda/docs/ortografia_basica.pdf

Cassany, Daniel. (1995). La cocina de la escritura. Barcelona, España: Anagrama.

Cassany, Daniel y Antonio García del Toro. (1999). Recetas para escribir. San Juan de Puerto Rico. Editorial Plaza Mayor.

Cassany, Daniel. (2006). Taller de textos. Leer, escribir y comentar en el aula. Barcelona, España: Paidós.

Cassany, Daniel. (2009). Enfoques didácticos para la enseñanza de la expresión escrita. Comunicación, Lenguaje y Educación, (6), 63-80. Recuperado el 13 de febrero de 2017, de https://ww w.upf.edu/pdi/dtf/daniel_cassany/enfoqes.htm

Cassany, Daniel (s/f)). Decálogo didáctico de la enseñanza de la composición. Recuperado el 22 de febrero de 2017, de https://www.upf.edu/pdi/dtf/daniel_cassany/decalogoco mposicion.pdf

Chartier, Anne-Marie (2016). Cierre. En el cuaderno de clase: las huellas del cotidiano leer y escribir. Flacso Virtual. Recuperado el 28 de febrero de 2017, de http://virtual.fla cso.org.ar/mod /book/view.php?id=124221\&chapterid=187071

C A White Robin. (2009). Writing Guide 2: Writing a Research Paper. 7th Edition. School of Law. University of Leicester. Recuperado el 15 de febrero de 2017, de https://ww w2.le.ac.uk/departments/law/current/writing-guide/Writing_Guide_Research_Paper_2009.pdf.

De Garay Adrian (s/f). Mis estudios y propuestas sobre los jóvenes universitarios mexicanos. Recuperado el 22 de febrero de 2017, de http://www.fundacionucol.org/ wp-content/uploads/2013/09/Estudios-ypropuestas-Adrian-de-Garay.pdf

Díaz-Barriga, Arceo Frida y Gerardo Hernández Rojas. (2002). Estrategias docentes para un aprendizaje significativo. Una interpretación constructivista. México. $2^{a}$. Ed. McGrawHill 
Fernández, G. y Carlino Paula. (2015). Leer y escribir en los primeros años de la universidad: Un estudio en Ciencias Veterinarias y Humanas de la UNCPBA. Cuadernos de Educación, 277-289. Recuperado el 28 de diciembre de 2015, de w ww://www.aacademica.org/paula.carlino/54 .pdf

Finocchio, Ana María. (2016). Las consignas de escritura en la escuela. En Cuando las consignas invitan a escribir. Recuperado el 20 de febrero de 2017, de http://virtual.f lacso.org.ar/mod/book/view.php?id=124261\&chapt erid=187511

Martínez, Ezquerro Aurora (2014). Talleres literarios. La Rioja. España: Universidad de la Rioja. Servicio de publicaciones. Recuperado el 15 de Febrero de 2017, de http: // biblioteca.unirioja.es/tfe_e/T FE000747.pdf

Martínez, M. J. (2010). Investigación etnográfica: Métodos de investigación en educación especial. México, México: Universidad Autónoma Metropolitana. Recuperado el 8 d e Febrero de 2017, de https://www.uam.es/personal_pdi/stmaria/jmurillo/Investiga cionEE/Presentacione s/Curso_10/I_Etnografica_Trabajo.pdf

Monzón, V. E. (2003). Estilo de citas y referencias de la American Psychological Association (APA). Caracas, Venezuela: Universidad Central de Venezuela. Recuperado el 22 de Diciembre de 2016, de http://documentslide.com/education/manual-del-apa-est ilo-de-citas-y-referencias-de-la-americanpsychological-association.html

Rincón, Castellanos Carlos Alberto. (s.f.). Unidad 14: La escritura III. Recuperado el 3 de E nero de 2017, de http://aprendeenlinea.udea.edu.co/boa/contenidos.php/cb10887d 80142488399661377b684b60/511/estilo/pn_vino/1/contenido/capitulos/Unidad14L aEscritura3.PDF

Ruíz, Zavala Alma, Beatriz López y Cervantes, Elisa Aguilar Funes y Tatiana Sorókina (Coordinadora). (2012). Géneros discursivos. Breve guía universitaria. México: Un iversidad Autónoma MetropolitanaXochimilco. Recuperado el 14 de Noviembre de 2016, de http://biblioteca.xoc.uam.mx/docs/ahistorico/generos_discursivos.pdf

Sanz, Hernández Alexia. (2005). El método biográfico en investigación social: Potencialidades y limitaciones de las fuentes orales y los documentos personales. Asclepio, 99-115. Recuperado el 8 de Febrero de 2017, de https://flacso.unah.ed u.hn/gestordocumentos/297

Troglia, María José y Elena Stapich. (2008). El taller de lectura. Un espacio para la autoconstrucción de lectores. En S. E. (Coordinadora), Textos, tejidos y tramas en el taller de lectura y escritura (pág. 163). Buenos Aires. Argentina: Ediciones Novedades Educativas. Recuperado el 15 de Febrero de 2017, de https://boo

ks.google.com.mx/books?id=NarkaxgvT9QC\&pg=PA19\&lpg=PA19\&dq=talleres+claire+boniface \&so urce=bl\&ots=RWjtqGwLTt\&sig=zM2TuAFD38ysgHxDrbiOYFiXU zA\&hl=es419\&sa=X\&ved=0ahUKEwjlzNWj6pLSAhUH92MKHWSXArQQ6AEIHjA $\mathrm{A} \# \mathrm{v}=$ onepage $\& \mathrm{q}=$ talleres $\% 20$ claire $\% 20$ boniface $\& \mathrm{f}$

Troglia, María José. (2008). Un taller y otros talleres. Vallas y trampolines a la hora de animar un taller de lectura y escritura. En Textos, tejidos y tramas en el taller de lectura y escritura. Buenos Aires. Argentina: Ediciones Novedades Educativas. Recuperado el 15 de Febrero de 2017, de https://books.google.com.mx/books?id

$=$ NarkaxgvT9QC \&pg=PA19\&lpg=PA19\&dq=talleres+claire+boniface $\&$ source $=$ bl $\&$ ots=RWjtqGwLTt\&sig=zM2TuAFD38ysgHxDrbiOYFiXUzA\&hl=es-419\&sa=X\&ved= 
0ahUKEwjlzNWj6pLSAhUH92MKHWSXArQQ6AEIHjAA\#v=onepage\&q=talleres\% 20claire\%20boniface\&f

Trujillo, Sáez Fernando. (2002). Los modelos textuales en la enseñanza de la escritura y la lectura. Europhoros, 11-22. Recuperado el 12 de enero de 2017, de http://fernand otrujillo.es/wp-content/uploads/ 2010/05/modesclec.pdf

Universidad Autónoma de Nayarit. Programa de estudios de la licenciatura en Químico Farmacobiólogo. Recuperado el 8 de febrero de 2017, de http://www.uan.edu.mx/e s/quimico-farmacobiologo

Universidad Autónoma de Nayarit. Programa de estudios de la licenciatura en Médico Cirujano. Recuperado el 8 de febrero de 2017, de http://www.uan.edu.mx/es/licenc iatura-medico-cirujano

Universidad Autónoma de Nayarit. Programa de estudios de la licenciatura en Enfermería. Recuperado el 8 de febrero de 2017, de http://www.uan.edu.mx/es/licenciatura-en-enfermeria

Zavala, Díaz Ana L. (2013). La "autobiografía" como una de las bellas artes. Revista de la Universidad de México (114), 87-88. Recuperado el 2 de Febrero de 2017, de http ://www.revistadelauniversidad.unam.mx/ojs_rum/index.php/rum/article/view/687 